Avto N. Tavkhelidze, Amiran Bibilashvili, Larissa Jangidze, Brian Billenberg and Gertrude F. Rempfer

\title{
Quantum interference depression in thin metal films with nanostructured surfaces
}

ABSTRACT. Quantum interference depression in thin metal films depends on the film internal structure and the width of the ridges. Grain size and the de Broglie wavelength distribution of the electrons determine the optimum ridge width to set the Fermi level. The Fermi energy increase due to QID can be used in devices using internal contact potential difference, such as Schottky diodes, and in metal/semiconductor ohmic contacts for power electronics.

Nanotechnology Perceptions 4 (2008) 25-28

Nonsubscribers purchase individual article 